\title{
Diagnosis and treatment options including stereotactic body radiation therapy (SBRT) for adrenal metastases
}

\author{
Rami W. Eldaya • Simon S. Lo • Arnold C. Paulino • \\ Angel I. Blanco • E. Brian Butler • Bin S. Teh
}

Received: 21 January 2012 / Accepted: 30 January 2012 / Published online: 28 February 2012

(C) Springer-Verlag 2012

\begin{abstract}
Adrenal metastasis is common in oncology but patients with this diagnosis are usually asymptomatic. The diagnosis can be challenging and delayed. Incidence, presenting symptoms and diagnostic modalities are reviewed. Various treatment options are also discussed, including open and laparoscopic surgery, local ablation (radiofrequency ablation, cryoablation, microwave thermal ablation, and chemical ablation) as well as radiation therapy. These treatment modalities were compared and contrasted in regards to their invasiveness, risks, side effects, outcomes, and patients' tolerance. Radiotherapy including stereotactic body radiation therapy (SBRT) is the only non-invasive approach, which is important as majority of the patients with adrenal metastases also receive systemic therapy, e.g., chemotherapy. The initial goal of conventional radiotherapy for metastatic adrenal lesion is for palliation which has been shown to be effective. With the advent of
\end{abstract}

R. W. Eldaya

University of Texas Medical Branch,

Galveston, TX, USA

S. S. Lo

Department of Radiation Oncology, Case Western Reserve

University, UH Seidman Cancer Center,

Cleveland, OH, USA

A. C. Paulino · A. I. Blanco • E. Brian Butler • B. S. Teh

Department of Radiation Oncology,

The Methodist Hospital, Cancer Center

and Research Institute,

Houston, TX, USA

B. S. Teh $(\bowtie)$

Department of Radiation Oncology,

The Methodist Hospital Cancer Center,

6565 Fannin, Ste\#DB1-077,

Houston, TX 77030, USA

e-mail: BTeh@tmhs.org technological advances in radiation oncology, e.g., imageguidance during radiation delivery, patient immobilization and patient re-positioning, tumor motion management, sophisticated treatment planning allowing rapid dose fall-off, accurate QA, etc., the clinical implementation of SBRT has been very successful. SBRT is defined as a "treatment method to deliver high dose of radiation to the target, utilizing either a single dose or a small number of fractions with a high degree of precision within the body". Adrenal metastasis, an example of oligometastases even from radioresistant primary, could be successfully treated with SBRT without significant toxicity as evidenced by various recently reported clinical trials. An additional advantage could be adrenal function preservation when compared to surgical intervention. The optimal total dose and fractionation scheme are yet to be determined. SBRT is an emerging non-invasive, safe, and effective treatment modality, and further research is warranted to define its role in the management of adrenal metastasis.

Keywords SBRT - Stereotactic body radiotherapy - Adrenal metastases $\cdot$ Diagnosis $\cdot$ Treatment options

\section{Introduction}

Adrenal gland is one of the most common organ sites of metastases. Metastasis is the second most common etiology of adrenal mass after adenoma [1]. Adrenal metastasis is a common finding on autopsies, reported to be between $13 \%$ and $35 \%$ [2-4]. The common primary malignancies which metastasize to the adrenals include cancers from lung (35\%$59 \%)[5,6]$, liver and bile duct (10\%) [5], breast $(30 \%-$ $40 \%$ ) [7], colon (14\%) [5], and kidneys (7\%-19\%) [8]. Other less common primary cancers include skin cancer (melanoma) [9, 10], esophageal cancer [5], stomach cancer 
[5], thyroid cancer (papillary [11], follicular, Hurthle [12]), anaplastic [13], prostate cancer [14], and gynecologic cancer [15].

The majority of adrenal metastases are carcinomas [5], that occur within a brief period after the diagnosis of the primary malignancy (latent period, 7 months), with only $2 \%$ of the cases metastasizing after 5 years [16]. The common prevalence of adrenal metastases, different diagnostic criteria, and different treatment modalities initiated this review. The purpose of this review is to look at the above-mentioned aspects and the emerging role of stereotactic body radiation therapy (SBRT) in the treatment of adrenal metastases.

\section{Clinical presentations}

The suspicion or detection of adrenal metastases solely based on symptomatic presentation is very rare since most adrenal metastases are silent or asymptomatic [5, 11]. Lam et al. studied data of 464 patients diagnosed with adrenal metastases for a 30 -year period and found that the $96 \%$ (444 patients) were asymptomatic at detection [5]. Anyhow, the presenting symptoms include adrenal insufficiency $[5,7,17]$, peritoneal hemorrhage [5], abdominal pain and pressure [10], and thrombosis of IVC [7, 15]. The fact that adrenal metastases are generally asymptomatic raises two questions:

1. What is the best way to detect adrenal metastases?

2. Is the goal of treatment palliative or curative?

\section{Detection and diagnosis}

The average age of patients with adrenal metastasis is 62 years. Even though biopsy remains the definitive diagnostic tool of adrenal metastases, imaging plays an important role [6]. Controversies still exist as to when a biopsy is needed. Harisinhgan et al. retrospectively examined 225 biopsies of adrenal masses performed from 1996 to 2001 with the purpose of testing the negative predictive value of negative biopsy [3]. Of the 225 biopsies in patients with primary non-adrenal malignancies and adrenal mass, 41 were determined negative and of these 13 did not have definitive diagnosis. A repeat biopsy was done and was negative for all 13, suggesting that the negative predictive value of CT guided biopsy was $100 \%$ [18]. In another study that examined 101 biopsies of adrenal mass, it was suggested that the positive predictive value of image-guided biopsy was $100 \%$, with the diagnosis obtained in $86 \%$ of cases. Among 72 patients with proven outcomes, image-guided biopsy had an accuracy of $96 \%$, sensitivity of $93 \%$, and a negative predictive value of $91 \%[19]$.
Complication rate of fine needle biopsy (FNA) is reported to be around $3 \%$. The complications include hemorrhage, infection, theoretical risk of tumor tract seeding, capsular disruption, and pheochromocytoma dynamic instability [20]. However, another study showed that complications such as pneumothorax, septicemia, and hemorrhage occurred in $8-13 \%$ of patients undergoing FNA [19]. The authors further suggested that since image-guided FNA biopsy cannot differentiate between an adrenal cortical carcinoma and an adrenal adenoma, but has sensitivity of $80 \%$ to $100 \%$ in detecting adrenal metastases, it should be reserved for adrenal masses suspicious for metastases [19].

CT has been used for the detection of adrenal metastases for more than 20 years. In one of the earlier reports in 1990, the authors studied the sensitivity and specificity of CT in detecting adrenal metastases from lung cancer in 91 patients. The results demonstrated that the sensitivity ranged from $20 \%$ to $41.4 \%$ and the specificity was much better ranging from $84.5 \%$ to $99.4 \%$ [21]. In recent years, special techniques such as high lipid density, enhancement after contrast, \% absolute enhancement washout, and \% relative enhancement washout have helped improve the specificity and sensitivity of CT [20]. Sensitivity and specificity have been reported to be as high as $100 \%$ with the use of $<60 \%$ absolute enhancement washout on CT [22].

With conventional MRI, the sensitivity and specificity have been reported to be less than those of CT [23]. The intensity signal on T2 and single intensity index, have increased the sensitivity and specificity tremendously. Fujiyoshi et al. examined MRI's capability to characterize adrenal tumors using chemical shift, and found that single intensity index (cut off $11.2 \%-16.5 \%$ ) was $100 \%$ accurate in determining metastatic adrenal lesions [24].

The use of 18F-FDG-PET in detecting systemic metastases and, specifically, adrenal metastases has shown very promising results. The role of PET imaging in cancer detection has been increasing in the last 10 years, and is now considered a part of the initial assessment of most cancer patients. PET offers an additional advantage over $\mathrm{CT}$ and MRI in determining the primary site of tumor and other possible metastatic sites [6]. Kumar et al. examined the sensitivity, specificity, and accuracy of recent studies using PET in detecting adrenal metastases, mainly from lung cancer, and reported $93-100 \%$ (sensitivity), 80-100\% (specificity), and 92-100\% (accuracy), respectively [6].

While biopsy has been historically the acceptable method in making the diagnosis of adrenal metastases, the advances made in imaging modalities in the last 20 years have challenged the role of biopsy. With sensitivity, specificity, and accuracy comparable to, if not better than, biopsy in detecting adrenal metastases, and unlike biopsy, no complications were reported. Currently, it is considered acceptable to use imaging as a diagnostic tool of adrenal metastases. 


\section{Treatment options}

Surgery

The presence of metastatic lesions in adrenals has been associated with dismal prognosis and short survival period. Before the 1960s, adrenal metastases were treated conservatively as a part of disseminated process with poor outcome. In the $1970 \mathrm{~s}$, surgeons started to manage this problem with adrenalectomy, showing good results, but it was a big and traumatic operation that required careful selection of patients [4]. However, the introduction of laparoscopy has rendered surgery the primary treating modality of adrenal metastases. Laparoscopic approach has been proven to be as effective as open surgery [4, 25], with the former associated

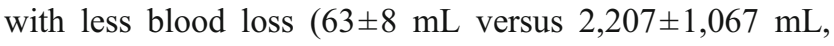
$P=0.05)$, a lower complication rate $(0 \%$ versus $63 \%, P=$ 0.009), and a shorter length of stay [26]. Contraindications to laparoscopic surgery include pregnancy, large tumors $(>8 \mathrm{~cm})$, or suspicion of primary invasive adrenocortical carcinoma on imaging. Other relative contraindications include morbid obesity, prior intra-abdominal surgery (adhesion was reported as the primary reason to convert a laparoscopic surgery to open surgery [27]), and large pheochromocytomas [7]. The majority of reports encourage surgical resection of adrenal mass measuring 5-6 cm [7] regardless of the cause, with the exception of nonfunctioning adrenal incidentaloma [28]. Surgical complication rate ranged between 4\% and 29.4\% [26, 27, 29, 30]. Complications include deep venous thrombosis, pulmonary embolism, stroke, pneumonia, hematoma, myocardiac infarction, atelectasis, bowel perforation, lung infection, and wound infection $[25,27,31]$. Recurrence rate was reported to be between $0-21 \%[26,30]$, and the 5-year survival between $22.5 \%$ and $47 \%[4,26]$.

Surgical approach to adrenal metastases has been studied extensively and established as the primary treatment modality. Surgery has been shown to be effective, especially when the primary cancer is controlled and there is no evidence of other metastases besides the adrenals [21].

\section{Percutaneous ablation}

Tumor ablation is defined as the direct application of chemical or thermal therapies to a tumor to achieve eradication or substantial destruction of tumor [32]. Percutaneous ablative approaches that have been described and used in the treatment of adrenal tumors include percutaneous radiofrequency ablation, cryoablation, microwave ablation, and chemical ablation. Local tumor ablation in the adrenal gland presents unique challenges because of the unique anatomy and physiology of adrenal gland.[33]. Therefore, at present, percutaneous ablation for adrenal neoplasms has primarily been applied in patients who are considered unresectable or poor surgical candidates, those who refuse surgery despite surgery being an option, or those who have multiple prior attempts at surgical debulking [33]. Risks and complications of adrenal ablation include bleeding, infection, hypertensive crisis, and in cases of thermal ablation, thermal injury to adjacent structures (including the kidneys, lungs, and pancreas) [33]. Of note, the reported studies involving local ablation had few patients and there was no prospective trials addressing these tumor ablative procedures.

\section{Radiation therapy}

The role of radiotherapy (XRT) in adrenal metastases was suggested more than 30 years ago. Earlier trials used XRT for palliative care of adrenal metastases and showed promising response. Soffen examined 16 patients treated between 1972 and 1988 for palliation of symptomatic adrenal metastases and found that 12 of 16 patients $(75 \%)$ showed partial to full symptomatic pain relief after treatment with XRT [34]. Soejima et al. examined 14 patients with adrenal metastases treated between 1985 and 1995 with XRT for symptomatic relief. Seven of eight patients presenting with pain $(87.5 \%)$ reported pain relief [35]. Short et al. examined 18 patients presenting with adrenal metastases in 1996 and reported complete pain relief after treatment with XRT in seven out of seven $(100 \%)$ patients presenting with pain [36].

The first report of the role of XRT as primary treatment modality for adrenal metastases was suggested in a case report in 1999 [37]. Since then, the role of XRT of adrenal metastases has been primarily reported in the form of sporadic case reports. There have been two larger series of patients with adrenal metastases treated with XRT. Oshiro et al. examined 19 patients with adrenal metastases from lung cancer treated with by radiation therapy, seven of whom received external beam radiation therapy and 11 receiving SBRT. The mean age was 63 years. They were all symptomatic. The overall 1-, 2- and 5-year survival rates for all patients were $56 \%, 33 \%$, and $22 \%$, respectively. More favorable prognosis was observed for patients with a metachronous metastasis, with overall 1-, 2-, and 5- year survival rates of $83 \%, 56 \%$, and $56 \%$, respectively. Tumor size (>3 cm), pathology, location (right or left adrenal gland), method of radiotherapy (with or without SBRT), and radiation dose (>50 Gy) all had no significant effect on overall survival [38].

Zeng et al. examined 22 patients with adrenal metastases from hepatocellular carcinoma, treated by XRT. Eleven of $14(78.6 \%)$ patients presenting with pain had complete pain relief after XRT and 2 patients had major relief of 


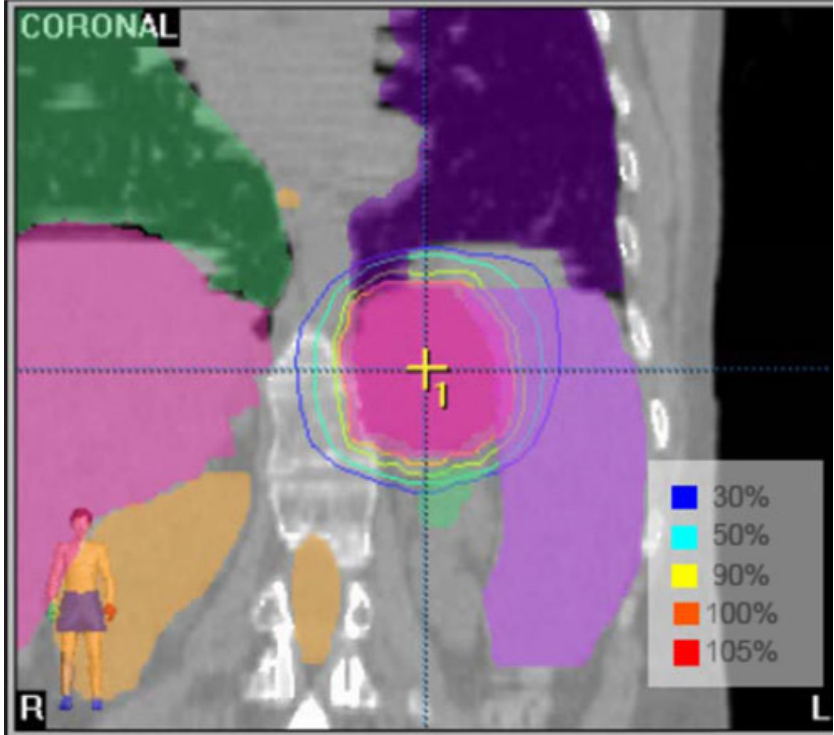

Fig. 1 SBRT treatment plan for left adrenal metastasis showing very tight isodose distribution with rapid fall-off in an effort to reduce the volume of surrounding critical normal tissues (kidney, bowels, stomach, spinal cord and liver) receiving high-dose radiation

symptoms, but still required analgesics. Median survival was 10 months with none dying from adrenal metastases complication, and partial response was observed in $73 \%$ of patients. Minor toxicity including nausea and vomiting was noted [39].

\section{Stereotactic body radiation therapy}

SBRT is defined by the American Society for Radiation Oncology and the American College of Radiology as a "treatment method to deliver a high dose of radiation to the target, utilizing either a single dose or a small number of fractions with a high degree of precision within the body" [40]. Some essential components needed for the clinical implementation of SBRT include patient immobilization and accurate reposition from simulation session to each treatment session, accounting for motion or tracking "moving target", fusion of various imaging studies, construction of tight dose distributions covering tumor with rapid fall-off the adjacent normal tissues, as well as the availability of imageguidance during treatment delivery [41].

The most common indications for SBRT are the treatment of non-metastatic primary cancer and oligometastases, with primary goal of treatment being local control [42]. Patients who are more likely to benefit from SBRT for their oligometastases include those who are younger, and those who have higher performance status, controlled primary tumor sites, metachronous occurrence of metastatic disease, limited number of metastases, and radioresistant histologies such as renal cell carcinoma [43]. The advantage of SBRT is its ability to deliver high doses of radiation to the planning treatment volume (PTV) while limiting the radiation dose to organs-at-risk [42]. Figure 1 shows a SBRT plan where a highly conformal prescription isodose line is created around the adrenal metastasis while keeping high-dose radiation away from the adjacent normal tissues such as the kidney and small bowels.

There have been four reports that studied the treatment of adrenal metastases using SBRT as shown in Table 1. The Japanese study by Oshiro et al. described above under the section "Radiation therapy" was included. Eleven patients were treated with SBRT. Casamassima et al. studied 48 patients from 2002 to 2009 with adrenal metastases that were treated with

Table 1 Clinical studies of SBRT for adrenal metastases

\begin{tabular}{|c|c|c|c|c|}
\hline & $\begin{array}{l}\text { Oshiro et al. (2011). } \\
\text { International Medical } \\
\text { Center of Japan [38] }\end{array}$ & $\begin{array}{l}\text { Casamassima et al. } \\
\text { (2011). University } \\
\text { of Florence [44] }\end{array}$ & $\begin{array}{l}\text { Chawla et al. (2009). } \\
\text { University of Rochester } \\
\text { Medical Center [45] }\end{array}$ & $\begin{array}{l}\text { Holy et al. } \\
\text { (2011). RWTH } \\
\text { Aachen [46] }\end{array}$ \\
\hline Number of patients & 11 & 48 & 30 & 18 \\
\hline Median age & 63 years & 62.7 years & 61.8 years & 61.5 years \\
\hline SBRT dose & Median 30 Gy (30-60) & Median 36 Gy & Median 40 Gy (16-50) & Median 40 Gy $(20-40)$ \\
\hline \# of fractions & $1-5$ fractions & $\begin{array}{l}3 \text { fraction } \\
\quad(\text { except } 8 \text { patients } 1)\end{array}$ & 4-10 fractions & 5 fractions \\
\hline Median follow-up & 10.1 month & 16.2 month & Until recurrence & 21 month \\
\hline Actuarial 1 year/2 year survival & $56 \% / 33 \%$ & $39.7 \% / 14.5 \%$ & $44 \% / 25 \%$ & $66.7 \%$ at 1 year \\
\hline 1 year distant control & & $9 \%$ & $13 \%$ & \\
\hline 1 year/2 year local control & $\begin{array}{l}79 \% \text { over } \\
\text { follow-up period }\end{array}$ & Both $90 \%$ & $55 \% / 27 \%$ & $\begin{array}{l}77 \% \text { for single } \\
\text { metastases }\end{array}$ \\
\hline Presenting symptoms & All asymptomatic & 4 pain/44 asymptomatic & 3 pain/27 asymptomatic & \\
\hline Toxicity & $\begin{array}{l}1 \text { grade } 2 \text { toxicity } \\
\text { peptic ulcer disease }\end{array}$ & 1 adrenal insufficiency & No patient had toxicity & $\begin{array}{l}6 \text { mild nausea, } \\
2 \text { peptic ulcers }\end{array}$ \\
\hline
\end{tabular}

Data compiled from source [38], [44], [45] and [46], respectively 
SBRT. On univariate and multivariate analyses, no significant factor (primary tumor, synchronous versus metachronous, unilateral and bilateral, oligometastatic disease, PTV, and number of fractions) emerged as an independent prognostic factor for overall survival, local control and distant control $(P>0.05)$ [44]. Among the four patients presenting with painful metastases, three were pain-free after SBRT and one had improved pain. Toxicity profile was excellent with only one patient having grade 2 adrenal insufficiency.

Chawla et al. examined 30 patients treated from 2001 to 2009. Fourteen patients with five or fewer metastatic lesions (including adrenal) underwent SBRT, with the intent of controlling all known sites of metastatic disease, and the other 16 patients underwent SBRT for palliation or prophylactic palliation of bulky adrenal metastases. Three patients with pain on presentation achieved complete pain relief after SBRT. None of the 30 patients treated with SBRT developed grade 2 or higher toxicity [45].

Holy et al. treated, between July 2002 and September 2009, 18 patients with adrenal metastases from non-small cell carcinoma with SBRT. An isolated adrenal metastasis was diagnosed in 13 patients, while 5 patients with multiple metastatic lesions had SBRT due to back pain. A median progression-free survival (PFS) of 4.2 months was obtained for the entire patient group, with a markedly increased PFS of 12 months in 13 patients with an isolated metastasis involving the adrenal gland. After a median follow-up of 21 months, 10 of 13 patients (77\%) with isolated adrenal metastasis achieved local control. In these patients, median overall survival was 23 months. Overall survival was excellent and comparable to that of surgical approach. The advantage of SBRT is that it is a non-invasive approach and can be performed as an outpatient procedure. Acute side effects were mild [46].

A recent case report using SBRT to treat a remaining contralateral adrenal metastasis from renal cell carcinoma described adrenal function preservation as an added benefit of SBRT [47]. The patient reported had nephrectomy and adrenalectomy at the initial diagnosis. In addition to successful SBRT treatment achieving complete response on pain and imaging, he also had normal adrenal function at more than 3 years follow-up, despite one remaining adrenal gland. Adrenal function preservation will not be possible with surgery in this case.

Historically, adrenal metastases have been treated primarily by surgery because of the challenges that organ motion and location of sensitive small bowels poses for radiation treatment [28]. However, with the advent of advanced technology, SBRT, which can account for organ motion, protect function of surrounding organs by highly sophisticated planning and treatment techniques, and offer comparable local control, can be considered as one of the treatment options for the treatment of adrenal metastases. Based on the emerging data from the literature, SBRT appears to be an effective non-invasive cancer treatment modality offering good palliation of symptoms and local control, with minimum toxicity [48-52]. Prospective trials are needed to better define the role of SBRT in this setting.

\section{Conclusion}

Adrenal glands are a common site harboring metastatic disease. The incidence, presenting symptoms, diagnostic modalities, and treatment options of adrenal metastases were reviewed. SBRT has emerged as a promising non-invasive treatment option for patient with adrenal metastasis. Limited data in the literature shows good symptomatic relief and local control. Adrenal function preservation may be an additional benefit with SBRT. More data from patients with longer follow-up are needed to define the role of SBRT for adrenal metastasis and it is best obtained in prospective clinical trials setting.

\section{References}

1. Uberoi J, Munver R (2009) Surgical management of metastases to the adrenal gland: open, laparoscopic, and ablative approaches. Curr Urol Rep 10:67-72

2. Abrams HL, Spiro R, Goldstein N (1950) Metastases in carcinoma: analysis of 1000 autopsied cases. Cancer 3:74-85

3. Harisinghani MG, Maher MM, Hahn PF et al (2002) Predictive value of benign percutaneous adrenal biopsies in oncology patients. Clin Radiol 57:898-901

4. Muth A, Persson F, Jansson S et al (2010) Prognostic factors for survival after surgery for adrenal metastasis. Eur J Surg Oncol 36:699-704

5. Lam KY, Lo CY (2002) Metastatic tumours of the adrenal glands: a 30-year experience in a teaching hospital. Clin Endocrinol (Oxf) 56:95-101

6. Kumar R, Xiu Y, Yu JQ et al (2004) 18F-FDG PET in evaluation of adrenal lesions in patients with lung cancer. J Nucl Med 45:20582062

7. Gittens PR Jr, Solish AF, Trabulsi EJ (2008) Surgical management of metastatic disease to the adrenal gland. Semin Oncol 35:172176

8. Foucar E, Dehner LP (1979) Renal cell carcinoma occurring with contralateral adrenal metastasis: a clinical and pathological trap. Arch Surg 114:959-963

9. Mittendorf EA, Lim SJ, Schacherer CW, Lucci A, Cormier JN, Mansfield PF, Gershenwald JE, Ross MI, Lee JE (2008) Melanoma adrenal metastasis: natural history and surgical management. Am J Surg 195:363-368

10. Rajaratnam A, Waugh J (2005) Adrenal metastases of malignant melanoma: characteristic computed tomography appearances. Australas Radiol 49:325-329

11. Malhotra G, Upadhye TS, Sridhar E et al (2010) Unusual case of adrenal and renal metastases from papillary carcinoma of thyroid. Unusual adrenal metastasis and abdominal carcinomatosis secondary to Hurthle cell carcinoma of the thyroid. Clin Nucl Med $35: 731-736$ 
12. Namwongprom S, Núñez RF, Yeung HW et al (2007) Unusual adrenal metastasis and abdominal carcinomatosis secondary to Hurthle cell carcinoma of the thyroid. Exp Clin Endocrinol Diabetes 115:694-696

13. Iagaru A, McDougall IR (2007) F-18 FDG PET/CT demonstration of an adrenal metastasis in a patient with anaplastic thyroid cancer. Clin Nucl Med 32:13-15

14. Kawahara T, Taguchi H, Yamagishi T et al (2009) A case of bilateral adrenal and pleural metastases from prostate cancer. Case Rep Oncol 2:217-219

15. Baron M, Hamou L, Laberge S et al (2008) Metastatic spread of gynaecological neoplasms to the adrenal gland: case reports with a review of the literature. Eur J Gynaecol Oncol 29:523-526

16. Mourra N, Hoeffel C, Duvillard P et al (2008) Adrenalectomy for clinically isolated metastasis from colorectal carcinoma: report of eight cases. Dis Colon Rectum 51:1846-1849

17. Bausewein C, Kühnbach R, Haberland B (2006) Adrenal insufficiency caused by bilateral adrenal metastases - a rare treatable cause for recurrent nausea and vomiting in metastatic breast cancer. Onkologie 229:203-205

18. Silverman SG, Mueller PR, Pinkney LP et al (1993) Predictive value of image-guided adrenal biopsy: analysis of results of 101 biopsies. Radiology 187:715-718

19. Moreira SG Jr, Pow-Sang JM (2002) Evaluation and management of adrenal masses. Cancer Control 9:326-334

20. Ilias I, Sahdev A, Reznek RH et al (2007) The optimal imaging of adrenal tumours: a comparison of different methods. Endocr Relat Cancer 14:587-599

21. Porte H, Siat J, Guibert B et al (2001) Resection of adrenal metastases from non-small cell lung cancer: a multicenter study. Ann Thorac Surg 71:981-985

22. Park BK, Kim CK, Kim B et al (2007) Comparison of delayed enhanced CT and 18F-FDG PET/CT in the evaluation of adrenal masses in oncology patients. J Comput Assist Tomogr 31:550-556

23. Valeri A, Bergamini C, Tozzi F et al (2011) A multi-center study on the surgical management of metastatic disease to adrenal glands. J Surg Oncol 103:400-405

24. Fujiyoshi F, Nakajo M, Fukukura Y et al (2003) Characterization of adrenal tumors by chemical shift fast low-angle shot MR imaging: comparison of four methods of quantitative evaluation. AJR Am J Roentgenol 180:1649-1657

25. Marangos IP, Kazaryan AM, Rosseland AR et al (2009) Should we use laparoscopic adrenalectomy for metastases? Scandinavian multicenter study. J Surg Oncol 100:43-47

26. Adler JT, Mack E, Chen H (2007) Equal oncologic results for laparoscopic and open resection of adrenal metastases. J Surg Res 140:159-164

27. Nakanishi S, Hori D, Maeda S et al (2006) Open adrenalectomy for adrenal metastases from lung cancer-usefulness and problem of laparoscopic adrenalectomy. Hinyokika Kiyo 52:757-760

28. Pacak K, Eisenhofer G, Grossman A (2007) The incidentally discovered adrenal mass. N Engl J Med 356:2005

29. Katoh N, Onimaru R, Sakuhara Y et al (2008) Real-time tumortracking radiotherapy for adrenal tumor. Radiother Oncol 87:418424

30. Tanvetyanon T, Robinson LA, Schell MJ et al (2008) Outcomes of adrenalectomy for isolated synchronous versus metachronous adrenal metastases in non-small-cell lung cancer: a systematic review and pooled analysis. J Clin Oncol 26:1142-1147

31. Sebag F, Calzolari F, Harding J et al (2006) Isolated adrenal metastasis: the role of laparoscopic surgery. World J Surg 30: 888-892

32. Simon CJ, Dupuy DE, Mayo-Smith WW (2005) Microwave ablation: principles and applications. Radiographics 25:S69-S83
33. Venkatesan AM, Locklin J, Dupuy DE et al (2010) Percutaneous ablation of adrenal tumors. Tech Vasc Interv Radiol 13:89-99

34. Soffen EM, Solin LJ, Rubenstein JH, Hanks GE (1990) Palliative radiotherapy for symptomatic adrenal metastases. Cancer 65: $1318-1320$

35. Soejima T, Hirota S, Hishikawa Y et al (1997) Radiation therapy for adrenal metastases Nippon Igaku Hoshasen Gakkai Zasshi 57:801-804

36. Short S, Chaturvedi A, Leslie MD (1996) Palliation of symptomatic adrenal gland metastases by radiotherapy. Clin Oncol 8:387389

37. Miyaji N, Miki T, Itoh Y et al (1999) Radiotherapy for adrenal gland metastasis from lung cancer: report of three cases. Radiat Med 17:71-75

38. Oshiro Y, Takeda Y, Hirano S et al (2011) Role of radiotherapy for local control of asymptomatic adrenal metastasis from lung cancer. Am J Clin Oncol 34:249-253

39. Zeng ZC, Tang ZY, Fan J et al (2005) Radiation therapy for adrenal gland metastases from hepatocellular carcinoma. Jpn J Clin Oncol 35:61-67

40. Potters L, Steinberg M, Rose C et al (2004) American Society for Therapeutic Radiology and Oncology; American College of Radiology. American Society for Therapeutic Radiology and Oncology and American College of Radiology practice guideline for the performance of stereotactic body radiation therapy Int J Radiat Oncol Biol Phys 60:1026-1032

41. Teh B, Bloch C, Galli-Guevara M et al (2007) The treatment of primary and metastatic renal cell carcinoma (RCC) with image guided stereotactic body radiation therapy (SBRT). Biomed Imaging Interv J $3:$ e6

42. Lo SS, Fakiris AJ, Chang EL et al (2010) Stereotactic body radiation therapy: a novel treatment modality. Nat Rev Clin Oncol 7:44-54

43. Lo SS, Fakiris AJ, Teh BS et al (2009) Stereotactic body radiation therapy for oligometastases. Expert Rev Anticancer Ther 9:621635

44. Casamassima F, Livi L, Masciullo S et al (2012) Stereotactic radiotherapy for adrenal gland metastases: University of Florence experience. Int J Radiat Oncol Biol Phys 82:919-923

45. Chawla S, Chen Y, Katz AW et al (2009) Stereotactic body radiotherapy for treatment of adrenal metastases. Int J Radiat Oncol Biol Phys 75:71-75

46. Holy R, Piroth M, Pinkawa M et al (2011) Stereotactic body radiation therapy (SBRT) for treatment of adrenal gland metastases from nonsmall cell lung cancer. Strahlenther Onkol 187:245-251

47. Eldaya RW, Paulino AC, Blanco AI, et al. (2012) Preservation of adrenal function after successful stereotactic body radiation therapy of metastatic renal cell carcinoma involving the remaining contralateral adrenal gland. Pract Radiat Oncol. doi:10.1016/j.prro.2011.11.002

48. Herfarth KK, Debus J, Lohr F et al (2001) Stereotactic single-dose radiation therapy of liver tumors: results of a phase I/II trial. J Clin Oncol 19:164-170

49. Lee MT, Kim JJ, Dinniwell R et al (2009) Phase I study of individualized stereotactic of liver metastases. J Clinical Oncol 27:1585-1591

50. Mendez Romero A, Wunderink W, Hussain SM et al (2006) Stereotactic body radiation therapy and metastatic liver tumors. A single institution phasenI-II study Acta Oncology 45:831-837

51. Rusthoven KE, Kavanagh BD, Burri SH et al (2009) Synchronous renal and adrenal masses: an analysis of 80 cases. Multi-institutional phase I/II trial of stereotactic body radiation therapy for lung metastases J Clin Oncol 27:1579-1584

52. Lo SS, Teh BS, Mayr NA et al (2010) Stereotactic body radiation therapy for oligometastases. Discov Med 10:247-254 